This item was submitted to Loughborough's Research Repository by the author.

Items in Figshare are protected by copyright, with all rights reserved, unless otherwise indicated.

\title{
From algebraic to analytic double product integrals
}

PLEASE CITE THE PUBLISHED VERSION

\section{LICENCE}

CC BY-NC-ND 4.0

\section{REPOSITORY RECORD}

Hudson, R.L.. 2019. "From Algebraic to Analytic Double Product Integrals". figshare. https://hdl.handle.net/2134/2734. 
This item was submitted to Loughborough's Institutional Repository (https://dspace.lboro.ac.uk/) by the author and is made available under the following Creative Commons Licence conditions.

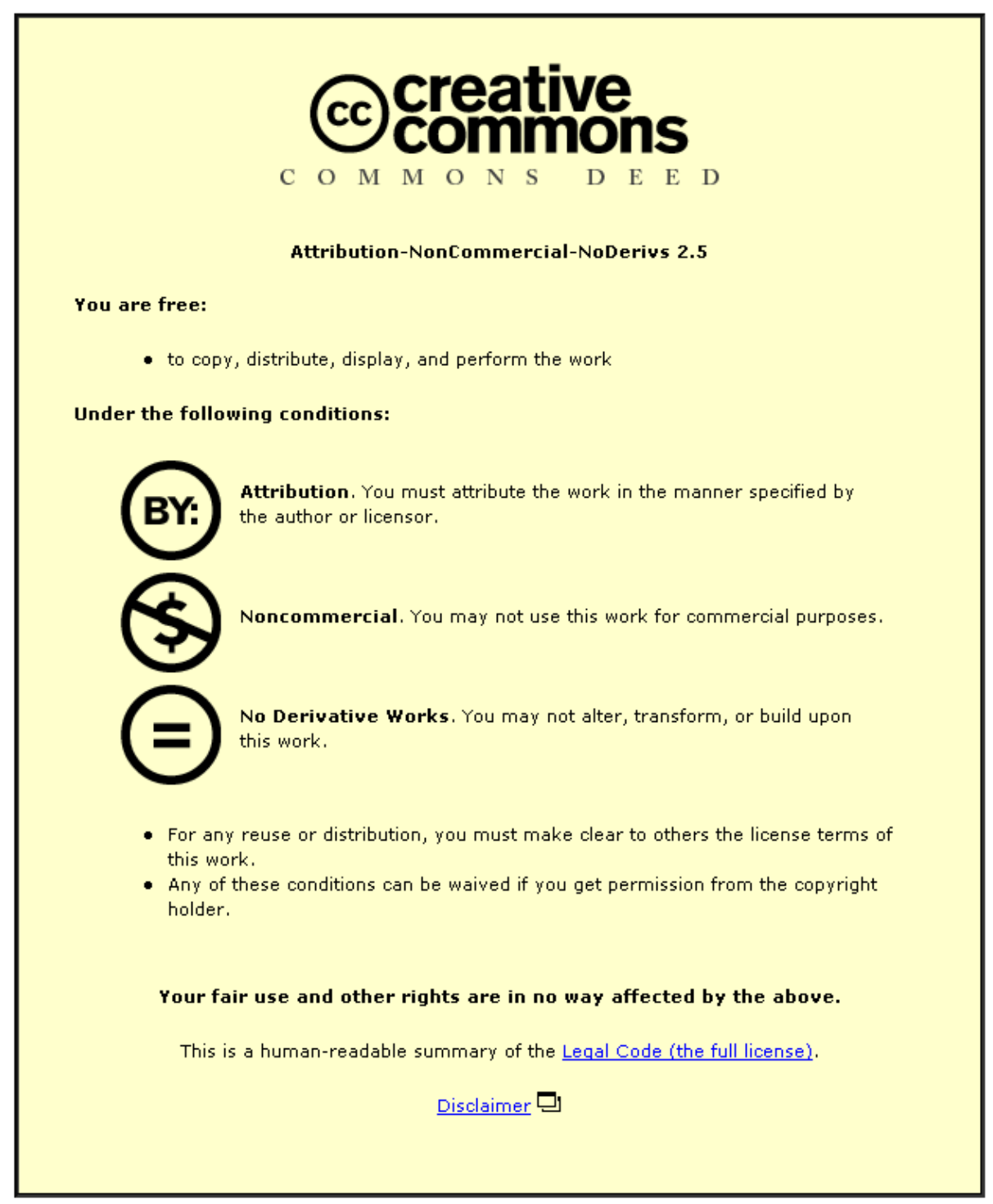

For the full text of this licence, please go to: http://creativecommons.org/licenses/by-nc-nd/2.5/ 


\title{
From algebraic to analytic double product integrals.
}

\author{
R L HUDSON
}

March 15, 2007

\begin{abstract}
The algebraic theory of double product integrals and particularly its role in the quantisation of Lie bialgebras is described. When the underlying associative algebra is that of the Itô differentials of quantum stochastic calculus such product integrals are formally represented as operators which are infinite sums of iterated integrals in Fock space. In this paper we describe some of the analytic problems encountered in making such sums rigourously meaningful, as well as the expected properties of such analytic double product integrals.

MRC classifucation: 81S 25.

Keywords: double productintegral, quantum stochastic inegral, iterated integral, quantum Yang-Baxter equation.
\end{abstract}

\section{INTRODUCTION}

An algebraic theory of double product integrals consisting of formal power series and thereby avoiding convergence questions has been developed. The original motivation for this theory was to construct a solution of the quantum Yang-Baxter equation from the datum of a generator in the form of a solution of the classical Yang-Baxter equation, thereby effecting a general quantisation procedure for quasitriangular Lie bialgebras in the sense of [3],[12]. It emerged [10] that in general the relevant double product integral was not generated directly by the corresponding cYBe solution but by a formal power series with vanishing zero-order coefficient of which the given solution was the first-order coefficient and whose higher-order coefficients are determined by a hierarchy of inhomogeneous linear equations previously considered in a related but distinct context in [2].

More recently the analytic double product integral generated by $d A^{\dagger} \otimes d A-d A \otimes$ $d A^{\dagger}$, where $A^{\dagger}$ and $A$ are the creation and annihilation processes of quantum stochastic calculus [14][13], was constructed in the form of a family of operators in Fock space satisfying quantum stochastic differential equations [7], as second quantisations of an explicitly evaluated double continuous product of infinitesimal rotations [8]. The existence of a general theory of quantum stochastic double product integrals in Fock space is impeded by the fact that one of the simplest such integrals, that generated by $z d \Lambda \otimes d \Lambda$ where $\Lambda$ is the conservation process of quantum stochastic calculus [14], is divergent in the sense that its matrix elements between exponential vectors have zero radius of convergence as power series in $z$. Another disincentive to constuction of such 
a general theory is awareness that the algebraic double products of most interest, those that furnish solutions of the qYBe, have generators which are themselves formal power series as noted above and thus give rise, when converted to Fock space objects using quantum stochastic calculus by replacing the formal parameter by a complex variable, to analytic quantum stochastic differential equations of a novel type for which the usual arguments for convergence of the series obtained by the Picard iterative method of solution do not apply without modification.

In this paper we consider some aspects of the transition from the algebraic, or "formal" in the language of some analysts, to an analytic theory of double product integrals in Fock space In Section 2 we recall so-called Itô Hopf algebra over an underlying associative algebra which reduces to the shuffle product Hopf algebra [2] when the underlying algebra is trivial, all products vanishing. In Section 3 we review the algebraic theory of double product integrals and their characterisation by their behaviour under the coproduct and in Section 4 their use to construct solutions of the quantum Yang-Baxter equation which are double product integrals and to use them to quantise Lie bialgebras. In Section 5 we show how, when the underlying algebra is that of Itô differentials in quantum stochastic calculus, the Itô Hopf algebra possesses a family of representations in Fock space in which homogeneous product tensors are mapped to iterated stochastic integrals, and through which the coproduct is related to the continuous tensor product splittings of Fock space (Theorem 5). Finally in Section 6 we consider some analytic problems arising when double product integrals are transferred to processes in Fock space by this mechanism.

\section{The ITÔ-Hopf ALGEBRA.}

Let there be given a not necessarily unital associative algebra $\mathcal{L}$ over the complex field $\mathbb{C}$. The vector space

$$
\mathcal{T}(\mathcal{L})=\mathbb{C} \oplus \mathcal{L} \oplus(\mathcal{L} \otimes \mathcal{L}) \oplus(\mathcal{L} \otimes \mathcal{L} \otimes \mathcal{L}) \oplus \cdots \oplus\left(\otimes^{n} \mathcal{L}\right) \oplus \cdots
$$

of all tensors over $\mathcal{L}$ becomes a Hopf algebra when equipped with the following structure.

- The associative product is defined by $\alpha \beta=\gamma=\left(\gamma_{0}, \gamma_{1}, \gamma_{2}, \ldots\right)$, where the $n$th rank component $\gamma_{n}$ of $\gamma$ is

$$
\gamma_{n}=\sum_{A \cup B=\{1,2, \ldots, n\}} \alpha_{|A|}^{A} \beta_{|B|}^{B},
$$

where the sum is over the $3^{n}$ decompositions of $\{1,2, \ldots, n\}$ into the union of two subsets, we use the place notation so that $\alpha_{|A|}^{A}$ indicates that the $|A|$ th rank component of $\alpha$ is to be placed in those copies of $\mathcal{L}$ within $\otimes^{n} \mathcal{L}$ which are labelled by elements of $A, \beta_{|B|}^{B}$ is defined analogously and if $A \cap B \neq \emptyset$ double occupancies of copies of $\mathcal{L}$ are reduced by multiplication in $\mathcal{L}$. 
- The unit element is $(1,0,0, \ldots)$.

- The coproduct $\Delta$ is defined by its action on homogeneous product tensors

$$
\Delta\left(L_{1} \otimes L_{2} \otimes \cdots L_{n}\right)=\sum_{j=0}^{n}\left(L_{1} \otimes L_{2} \otimes \cdots L_{j}\right) \bigotimes\left(L_{j+1} \otimes L_{j+2} \otimes \cdots L_{n}\right) .
$$

- The counit $\varepsilon$ is defined by

$$
\varepsilon\left(\alpha_{0}, \alpha_{1}, \alpha_{2}, \ldots\right)=\alpha_{0}
$$

- The antipode $S$ acts on homogeneous product tensors embedded in $\mathcal{T}(\mathcal{L})$ as

$$
S(L)=-L ; S\left(L_{1} \otimes L_{2} \otimes \cdots L_{n}\right)=(-1)^{n}\left(L_{n} \otimes L_{n-1} \otimes \cdots L_{1}\right)+\text { lower rank terms. }
$$

Note that when $\mathcal{L}$ is trivial, all products vanishing, the sum defining the product reduces to one over pairs of disjoint subsets whose union $\{1,2, \ldots, n\}$, and the Hopf algebra is then the shuffle product Hopf algebra of [2]. The proof that it is indeed a Hopf algebra for general $\mathcal{L}$, in particular that the coproduct remains multiplicative for the modified product, is in [9].

The kernel $\mathcal{K}(\varepsilon)$ of the counit consists of all tensors with vanishing zero-rank component. The map

$$
\varepsilon_{1}: \mathcal{K}(\varepsilon) \ni\left(0, \alpha_{1}, \alpha_{2}, \ldots\right) \mapsto \alpha_{1} \in \mathcal{L}
$$

is a homomorphism of associative algebras from $\mathcal{K}(\varepsilon)$ to $\mathcal{L}$.

Let $\mathcal{U}(\mathcal{L})$ denote the universal enveloping algebra of the Lie algebra got by equipping $\mathcal{L}$ with the commutator product. Then the map

$$
\phi: \mathcal{L} \ni L \mapsto(0, L, 0,0, \ldots) \in \mathcal{T}(\mathcal{L})
$$

is a Lie algebra homomorphism from the Lie algebra $\mathcal{L}$ to $\mathcal{T}(\mathcal{L})$ when the latter is also equipped with the commutator Lie bracket. Denote its universal extension by $\Phi$.

Theorem 1. $\Phi$ is an isomorphism of Hopf algebras from $\mathcal{U}(\mathcal{L})$ onto the sub-Hopf algebra of $\mathcal{T}(\mathcal{L})$ consisting of symmetric tensors.

Proof. That $\Phi$ is an isomorphism of unital associative algebras is proved in [11] in the context that $\mathcal{L}$ is the algebra of Itô differentials of quantum stochastic calculus, and the proof does not depend on this context. To prove that

$$
\Delta \Phi=(\Phi \otimes \Phi) \Delta
$$


where on the right hand side $\Delta$ is the coproduct of $\mathcal{U}(\mathcal{L})$, which is the universal extension of the Lie algebra homomorphism

$$
\mathcal{L} \ni L \mapsto L \otimes 1_{\mathcal{U}(\mathcal{L})}+1_{\mathcal{U}(\mathcal{L})} \otimes L \in \mathcal{U}(\mathcal{L}) \otimes \mathcal{U}(\mathcal{L})
$$

note first that (3) holds for actions on arbitrary $L \in \mathcal{L} \subset \mathcal{U}(\mathcal{L})$ by (2) and (1). That (3) holds in full generality now follows from the facts that $\mathcal{U}(\mathcal{L})$ is generated as an algebra by $\mathcal{L}$ and that both coproducts and the maps $\Phi$ and $\Phi \otimes \Phi$ are multiplicative. That $\Phi$ intertwines antipodes follows similarly from the facts that it does so by definition when applied to each $L \in \mathcal{L} \subset \mathcal{U}(\mathcal{L})$ (whose antipode in $\mathcal{U}(\mathcal{L})$ is $-L$ ), that $\Phi$ is multiplicative and that antipodes are antimultiplicative.

We introduce the (right) differential map $d$ from $\mathcal{T}(\mathcal{L})$ to $\mathcal{T}(\mathcal{L}) \otimes \mathcal{L}$ by linear extension of its action on homogeneous product tensors

$$
d\left(1_{\mathcal{T}(\mathcal{L})}\right)=0, d\left(L_{1} \otimes L_{2} \otimes \cdots L_{n}\right)=\left(L_{1} \otimes L_{2} \otimes \cdots L_{n-1}\right) \otimes L_{n} ;
$$

equivalently, for arbitrary $T$,

$$
d(T)=\left(\operatorname{id}_{\mathcal{T}(\mathcal{L})} \otimes \varepsilon_{1}\right)\left(\Delta(T)-T \otimes 1_{\mathcal{T}(\mathcal{L})}\right) .
$$

Here the right hand side is well defined because $\Delta(T)-T \otimes 1_{\mathcal{T}(\mathcal{L})} \in \mathcal{T}(\mathcal{L}) \otimes \mathcal{K}(\varepsilon)$, as is clear by linear extension of the case when $T$ is a homogeneous product tensor which follows from (1).

Note that $d$ maps $\mathcal{U}(\mathcal{L})$ to $\mathcal{U}(\mathcal{L}) \otimes \mathcal{L}$.

\section{Algebraic theory of Double product integrals.}

Let $\mathcal{A}$ be a unital associative algebra which we call the system algebra and let $k[h] \in$ $h(\mathcal{A} \otimes \mathcal{L})[[h]]$ be a formal power series with vanishing zero-order term and coefficients in the tensor product algebra $\mathcal{A} \otimes \mathcal{L}$. We define the forward and backward product integrals generated by $k[h]$, using place notation, as the formal power series with coefficients in $\mathcal{A} \otimes \mathcal{T}(\mathcal{L})=\bigoplus_{n=0}^{\infty} \mathcal{A} \otimes\left(\otimes^{n} \mathcal{L}\right)$

$$
\begin{aligned}
& \vec{\prod}(1+d k[h])=\sum_{n=0}^{\infty} k[h]^{0,1} k[h]^{0,2} \ldots k[h]^{0, n} \\
& \overleftarrow{\prod}(1+d k[h])=\sum_{n=0}^{\infty} k[h]^{0, n} k[h]^{0, n-1} \ldots k[h]^{0,1}
\end{aligned}
$$

where the zero-order term of the series is defined to be $1_{\mathcal{A} \otimes \mathcal{T}(\mathcal{L})}$ in each case. Equivalently they are the solutions $X[h]$ and $Y[h]$ of the algebraic stochastic differential equations

$$
\begin{aligned}
& \left(\operatorname{id}_{\mathcal{A}} \otimes d\right) X[h]=X[h]^{1,2} k[h]^{1,3},\left(\mathrm{id}_{\mathcal{A}} \otimes \varepsilon\right) X[h]=1_{\mathcal{A}} \\
& \left(\operatorname{id}_{\mathcal{A}} \otimes d\right) Y[h]=k[h]^{1,3} Y[h]^{1,2},\left(\mathrm{id}_{\mathcal{A}} \otimes \varepsilon\right) Y[h]=1_{\mathcal{A}} .
\end{aligned}
$$


If $\mathcal{A}$ is non-unital we may still define the decapitated product integrals $\widehat{\vec{\prod}}(1+d k[h])$ and $\widehat{\overparen{\Pi}}(1+d k[h])$ either by omitting the zero-order term in the series expansions or as the solutions $\hat{X}[h]$ and $\hat{Y}[h]$ of

$$
\begin{aligned}
& \left(\operatorname{id}_{\mathcal{A}} \otimes d\right) \hat{X}[h]=\left(\hat{X}[h]^{1,2}+1^{2} \mathcal{T}(\mathcal{L})\right) k[h]^{1,3},\left(\operatorname{id} \mathcal{A}_{\mathcal{A}} \otimes \varepsilon\right) \hat{X}[h]=0_{\mathcal{A}} \\
& \left(\operatorname{id}_{\mathcal{A}} \otimes d\right) \hat{Y}[h]=k[h]^{1,3}\left(\hat{Y}[h]^{1,2}+1^{2} \mathcal{T}(\mathcal{L})\right),\left(\operatorname{id}_{\mathcal{A}} \otimes \varepsilon\right) \hat{Y}[h]=0_{\mathcal{A}} .
\end{aligned}
$$

Note that these belong to $h(\mathcal{L} \otimes \mathcal{T}(\mathcal{L}))[[h]]$. Analogous definitions of the product integrals $\vec{\Pi}(1+d k[h]), \overleftarrow{\Pi}(1+d k[h]), \widehat{\vec{\Pi}}(1+d k[h])$ and $\widehat{\Pi}(1+d k[h])$, now belonging to $(\mathcal{T}(\mathcal{L}) \otimes \mathcal{A})[[h]]=\left(\bigoplus_{n=0}^{\infty}\left(\otimes^{n} \mathcal{L}\right) \otimes \mathcal{A}\right)[[h]]$ and to $h(\mathcal{T}(\mathcal{L}) \otimes \mathcal{A})[[h]]$ respectively, hold for a generator $k[h] \in h(\mathcal{L} \otimes \mathcal{A})[[h]]$.

Now let $r[h] \in h(\mathcal{L} \otimes \mathcal{L})[[h]]$ be a formal power series with vanishing zero-order term and coefficients in $\mathcal{L} \otimes \mathcal{L}$. Taking the first copy of $\mathcal{L}$ in $\mathcal{L} \otimes \mathcal{L}$ as the system algebra we may form the decapitated product integral $\overleftarrow{\prod}(1+d r[h]) \in h(\mathcal{L} \otimes \mathcal{T}(\mathcal{L}))[[h]]$. Using this as a generator we may then form $\vec{\prod}\left(1+\widehat{\prod}(1+d r[h])\right)$. Alternatively, taking the second copy of $\mathcal{L}$ as the system algebra we may form first $\widehat{\vec{\prod}}(1+d r[h]) \in h(\mathcal{T}(\mathcal{L}) \otimes \mathcal{L})[[h]]$ and then using this as a generator, $\overleftarrow{\prod}\left(1+\widehat{\vec{\prod}}(1+d r[h])\right)$. In fact $[9]$ these two constructions yield one and the same element of $(\mathcal{T}(\mathcal{L}) \otimes \mathcal{T}(\mathcal{L}))[[h]]$ which we define to be the forward-backward double product integral

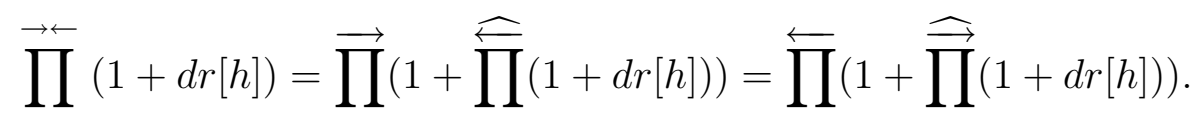

The double product integrals $\stackrel{\leftarrow}{\Pi}(1+d r[h]), \vec{\prod}(1+d r[h])$ and $\stackrel{\leftarrow}{\prod}(1+d r[h])$ are defined similarly by appropriate reversals of arrows.

Double product integrals can be characterised as elements $(\mathcal{T}(\mathcal{L}) \otimes \mathcal{T}(\mathcal{L}))[[h]]$ of by their behaviour under the actions of the coproduct [9]. For example the forwardbackward integral is characterised as follows

Theorem 2. Let $W[h]$ be a nonzero element of $(\mathcal{T}(\mathcal{L}) \otimes \mathcal{T}(\mathcal{L}))[[h]]$. Then the following are equivalent:

- There exists $r[h] \in h(\mathcal{L} \otimes \mathcal{L})[[h]]$ such that $W[h]=\vec{\prod}(1+d r[h])$.

$$
\begin{aligned}
\left(\Delta \otimes \operatorname{id}_{\mathcal{T}(\mathcal{L})}\right) W[h] & =W[h]^{1,3} W[h]^{2,3} \\
\left(\operatorname{id}_{\mathcal{T}(\mathcal{L})} \otimes \Delta\right) W[h] & =W[h]^{1,3} W[h]^{1,2} .
\end{aligned}
$$


Similar characterisations for otherwise directed double product integrals are found by reversing arrows in the first condition and reversing products in the second.

For $r[h] \in h(\mathcal{L} \otimes \mathcal{L})[[h]]$ the quasi-inverse is the unique element $r^{\prime}[h]$ of $h(\mathcal{L} \otimes \mathcal{L})[[h]]$ such that

$$
r[h]+r^{\prime}[h]+r[h] r^{\prime}[h]=r^{\prime}[h]+r[h]+r^{\prime}[h] r[h]=0 .
$$

The following theorem is proved in [10].

Theorem 3. The inverse of $\vec{\Pi}(1+d r[h])$ is $\stackrel{\leftarrow}{\Pi}\left(1+d r^{\prime}[h]\right)$ where $r^{\prime}[h]$ is the quasi-inverse of $r[h]$.

\section{The QUANTUm YANG-BAXter EQUATION AND QUANTISATION OF LiE BIALGEBRAS.}

In $[10]$ the following theorem is proved.

Theorem 4. A necessary and sufficient condition that $R[h]=\vec{\prod}(1+d r[h])$ satisfy the quantum Yang-Baxter equation

$$
R[h]^{1,2} R[h]^{1,3} R[h]^{2,3}=R[h]^{2,3} R[h]^{1,3} R[h]^{1,2}
$$

in $(\mathcal{T}(\mathcal{L}) \otimes \mathcal{T}(\mathcal{L}) \otimes \mathcal{T}(\mathcal{L})[[h]]$ is that $r[h]$ satisfy

$$
\begin{aligned}
& r[h]^{1,2} r[h]^{1,3}+r[h]^{1,2} r[h]^{2,3}+r[h]^{1,3} r[h]^{2,3}+r[h]^{1,2} r[h]^{1,3} r[h]^{2,3} \\
= & r[h]^{1,3} r[h]^{1,2}+r[h]^{2,3} r[h]^{1,2}+r[h]^{2,3} r[h]^{1,3}+r[h]^{2,3} r[h]^{1,3} r[h]^{1,2}
\end{aligned}
$$

in $(\mathcal{L} \otimes \mathcal{L} \otimes \mathcal{L})[[h]]$.

Note that the condition (4) is equivalent to $1+d r[h]$ satisfying the quantum YangBaxter equation in the algebra $\left(\mathcal{L}^{\prime} \otimes \mathcal{L}^{\prime} \otimes \mathcal{L}^{\prime}\right)[[h]]$ where $\mathcal{L}^{\prime}$ is got by adjoining a unit element 1 to $\mathcal{L}$.

Equating coefficients of powers of $h$ in (4) we obtain, for the lowest order coefficient $r_{1} \in \mathcal{L} \otimes \mathcal{L}$

$$
r_{1}^{1,2} r_{1}^{1,3}+r_{1}^{1,2} r_{1}^{2,3}+r_{1}^{1,3} r_{1}^{2,3}=r_{1}^{1,3} r_{1}^{1,2}+r_{1}^{2,3} r_{1}^{1,2}+r_{1}^{2,3} r_{1}^{1,3}
$$

that is, $r_{1}$ satisfies the classical Yang-Baxter equation

$$
C Y B\left(r_{1}\right) \equiv\left[r_{1}^{1,2}, r_{1}^{1,3}\right]+\left[r_{1}^{1,2}, r_{1}^{2,3}\right]+\left[r_{1}^{1,3}, r_{1}^{2,3}\right]=0
$$

while for higher order coefficients $C Y B\left(r_{n}\right)$,is equal to a polynomial expression in $r_{1}^{j . k}, r_{2}^{j . k}, \ldots r_{n-1}^{j . k},(j, k)=(1,2),(1,3),(2,3)$. It can be shown [2] that, for given $r_{1}$ satisfying $C Y B\left(r_{1}\right)=0$, the resulting hierarchy of inhomogeneous linear equations 
for the successive higher order equations has solutions so that a corresponding solution $r[h]$ of (4) can be constructed. For example, if $d r_{1}=d \Lambda \otimes d A-d A \otimes d \Lambda$. where $d \Lambda$ and $d A$ are the Itô differentials of the conservation and annihilation processes $\Lambda$ and $A$ of quantum stochastic calculus satisfying $(d \Lambda)^{2}=d \Lambda, d A d \Lambda=d A, d \Lambda d A=(d A)^{2}=0$, then it may be verified, firstly, that $C Y B\left(d r_{1}\right)=0$, and, secondly, that the hierarchy is satisfied by taking each $d r_{n}=0$ for $n \geq 2$. Hence $\vec{\prod}(1+h(d \Lambda \otimes d A-d A \otimes d \Lambda))$ satisfies the quantum Yang Baxter equation.

A solution of the quantum Yang-Baxter equation of form $R[h]=\vec{\prod}(1+d r[h])$ may be used to construct a deformation coproduct

$$
\Delta[h](\alpha[h])=R[h] \Delta(\alpha[h]) R[h]^{-1}
$$

in particular for the algebra $\mathcal{A}[[h]]$ of formal power series with coefficients in $\mathcal{T}(\mathcal{L})$ whose zero order coefficients lie in the symmetric subalgebra $\mathcal{S}(\mathcal{L}) \subset \mathcal{T}(\mathcal{L})$. Indeed it is clear that the map $\Delta[h]$ defined by this equation inherits multiplicativity from $\Delta$, while its coassociativity follows from that of $\Delta$ together with the quantum YangBaxter equation for $R[h]$ and $R[h]^{-1}$. The resulting deformation Hopf algebra provides a simple method of quantisation of the Lie bialgebra got by equipping the Lie algebra $\mathcal{L}$ with the cobracket $\delta$ which is the infinitesimal of $\Delta[h]$, given by

$$
\begin{aligned}
\delta(L) & =\frac{1}{h}\left(\Delta[h](L)-\Delta_{\mathrm{op}}[h](L)\right)+o(h) \\
& =(L \otimes 1+1 \otimes L)\left(r_{1}-\tau_{(2.1)} r_{1}\right)-\left(r_{1}-\tau_{(2.1)} r_{1}\right)(L \otimes 1+1 \otimes L) .
\end{aligned}
$$

Here $\Delta_{\text {op }}[h]$ denotes the opposite coproduct, $\Delta_{\text {op }}[h]=\tau_{(2.1)} \Delta[h]$ where (for an arbitrary vector space $\mathcal{V}) \tau_{(2.1)}$ is the flip linear transformation in $\mathcal{V} \otimes \mathcal{V}$ which exchanges the components of product tensors.

Thus the Lie bialgebra $\mathcal{L}$ is the quasitriangular Lie bialgebra generated by the solution $r_{1}$ of the classical Yang Baxter equation [3],[12] and we have an effective general method for the quantisation of such Lie bialgebras.

\section{Representation and COREpresentation properties in Fock SpaCe.}

In this section we take $\mathcal{L}$ to be the algebra $\mathcal{L}=\mathbb{C}\left\langle d \Lambda_{\alpha}^{\beta} ; \alpha, \beta=0,1,2, \ldots, N\right\rangle$ of Itô differentials of $N$-dimensional quantum stochastic calculus, with product defined by

$$
d \Lambda_{\alpha}^{\beta} d \Lambda_{\delta}^{\gamma}=\hat{\delta}_{\alpha}^{\gamma} d \Lambda_{\delta}^{\beta}
$$

where $\hat{\delta}_{\alpha}^{\gamma}=1$ if $\alpha=\gamma \neq 0$ and $\hat{\delta}_{\alpha}^{\gamma}=0$ otherwise. The corresponding processes consist of operators in the Fock space $\mathcal{F}\left(L^{2}\left(\mathbb{R}_{+} ; \mathbb{C}^{N}\right)\right)$ over the Hilbert space 
$L^{2}\left(\mathbb{R}_{+} ; \mathbb{C}^{N}\right)=L^{2}\left(\mathbb{R}_{+}\right) \otimes \mathbb{C}^{N}$ acting on the exponential domain, with matrix elements between exponential vectors given by

$$
\left\langle e(f), \Lambda_{\alpha}^{\beta}(t) e(g)\right\rangle=\int_{0}^{t} f^{\beta}(s) g_{\alpha}(s) d s\langle e(f), e(g)\rangle
$$

where for $f=\left(f_{1}, f_{2}, \ldots, f_{N}\right)^{T} \in L^{2}\left(\mathbb{R}_{+} ; \mathbb{C}^{N}\right), f_{0} \equiv 1$ and $f^{\alpha}(s)=\bar{f}_{\alpha}(s)$.

The iterated stochastic integral $\int_{a<s_{1}<s_{2}<\cdots<s_{n}<b} d \Lambda_{\alpha_{1}}^{\beta_{1}}\left(s_{1}\right) d \Lambda_{\alpha_{2}}^{\beta_{2}}\left(s_{2}\right) \ldots d \Lambda_{\alpha_{n}}^{\beta_{n}}\left(s_{n}\right)$ between $a<b \in \mathbb{R}_{+}$may be defined by its matrix elements between exponential vectors

$$
\begin{aligned}
& \left\langle e(f), \int_{a<s_{1}<s_{2}<\cdots<s_{n}<b} d \Lambda_{\alpha_{1}}^{\beta_{1}}\left(s_{1}\right) d \Lambda_{\alpha_{2}}^{\beta_{2}}\left(s_{2}\right) \ldots d \Lambda_{\alpha_{n}}^{\beta_{n}}\left(s_{n}\right) e(g)\right\rangle \\
= & \int_{a<s_{1}<s_{2}<\cdots<s_{n}<b} f^{\beta_{1}}\left(s_{1}\right) g_{\alpha_{1}}\left(s_{1}\right) f^{\beta_{2}}\left(s_{2}\right) g_{\alpha_{2}}\left(s_{2}\right) \ldots f^{\beta_{n}}\left(s_{n}\right) g_{\alpha_{n}}\left(s_{n}\right)\langle e(f), e(g)\rangle .
\end{aligned}
$$

The action of its adjoint on exponential vectors is obtained by exchanging $\alpha$ and $\beta$ indices while conserving the order of the terms in the integrator;

$$
\begin{aligned}
& \left(\int_{a<s_{1}<s_{2}<\cdots<s_{n}<b} d \Lambda_{\alpha_{1}}^{\beta_{1}}\left(s_{1}\right) d \Lambda_{\alpha_{2}}^{\beta_{2}}\left(s_{2}\right) \ldots d \Lambda_{\alpha_{n}}^{\beta_{n}}\left(s_{n}\right)\right)^{\dagger} \\
= & \int_{a<s_{1}<s_{2}<\cdots<s_{n}<b} d \Lambda_{\beta_{1}}^{\alpha_{1}}\left(s_{1}\right) d \Lambda_{\beta_{2}}^{\alpha_{2}}\left(s_{2}\right) \ldots d \Lambda_{\beta_{n}}^{\alpha_{n}}\left(s_{n}\right) .
\end{aligned}
$$

For $a<b \in \mathbb{R}_{+}$and $X \in \mathcal{T}(\mathcal{L})$ we define an operator $J_{a}^{b}(X)$ on the exponential domain by linear extension of the prescription

$$
J_{a}^{b}\left(d \Lambda_{\alpha_{1}}^{\beta_{1}} \otimes d \Lambda_{\alpha_{2}}^{\beta_{2}} \otimes \ldots \otimes d \Lambda_{\alpha_{n}}^{\beta_{n}}\right)=\int_{a<s_{1}<s_{2}<\cdots<s_{n}<b} d \Lambda_{\alpha_{1}}^{\beta_{1}}\left(s_{1}\right) d \Lambda_{\alpha_{2}}^{\beta_{2}}\left(s_{2}\right) \ldots d \Lambda_{\alpha_{n}}^{\beta_{n}}\left(s_{n}\right) .
$$

Then the map $J_{a}^{b}$ is a weak representation of the algebra $\mathcal{T}(\mathcal{L})$ in the sense that [11]

Theorem 5. For arbitrary $X, Y \in \mathcal{T}(\mathcal{L})$ and exponential vectors $e(f), e(g)$

$$
\left\langle\left(J_{a}^{b}(X)\right)^{\dagger} e(f), J_{a}^{b}(Y) e(g)\right\rangle=\left\langle e(f), J_{a}^{b}(X Y) e(g)\right\rangle .
$$

Note that each operator belonging to the range of $J_{a}^{b}$ is biadapted to the interval ]$a, b]$ in the sense that it takes the form of a double ampliation $\operatorname{id}_{\left.\mathcal{F}\left(L^{2}\left(\mathbb{R}_{+} ;\right] 0, a\right] ; \mathbb{C}^{N}\right)} \otimes P_{a}^{b}$ $\otimes \operatorname{id}_{\mathcal{F}\left(L^{2}\left(\mathbb{R}_{+} ; b, \infty\right] ; \mathbb{C}^{N}\right)}$ in the joint splitting at $a$ and $b$.

$$
\begin{aligned}
& \mathcal{F}\left(L^{2}\left(\mathbb{R}_{+} ; \mathbb{C}^{N}\right)\right) \\
= & \left.\left.\left.\mathcal{F}\left(L^{2}\left(\mathbb{R}_{+} ;\right] 0, a\right] ; \mathbb{C}^{N}\right) \otimes \mathcal{F}\left(L^{2}\left(\mathbb{R}_{+} ;\right] a, b\right] ; \mathbb{C}^{N}\right) \otimes \mathcal{F}\left(L^{2}\left(\mathbb{R}_{+} ;\right] b, \infty\right] ; \mathbb{C}^{N}\right)
\end{aligned}
$$


where $P_{a}^{b}$ is an operator on the exponential domain in $\left.\mathcal{F}\left(L^{2}\left(\mathbb{R}_{+} ;\right] a, b\right] ; \mathbb{C}^{N}\right)$.

The following theorem must be well-known to many quantum probabilists but a formal proof seems to be lacking in the literature, so one is given here. For $a<b<$ $c \in \mathbb{R}_{+}$we denote by $F_{a}^{c}(b)$ the splitting isomorphism from $\left.\mathcal{F}\left(L^{2}\left(\mathbb{R}_{+} ;\right] a, c\right] ; \mathbb{C}^{N}\right)$ to $\left.\left.\mathcal{F}\left(L^{2}\left(\mathbb{R}_{+} ;\right] a, b\right] ; \mathbb{C}^{N}\right) \otimes \mathcal{F}\left(L^{2}\left(\mathbb{R}_{+} ;\right] b, c\right] ; \mathbb{C}^{N}\right)$ under which each exponential vector $e(f)$ is mapped to the product vector $e\left(\left.f\right|_{] a, b]}\right) \otimes e\left(\left.f\right|_{] b, c]}\right)$. We identify elements of the range of $J_{a}^{b}$ with the operators of which they are the double ampliations corresponding to the double splitting (5).

Theorem 6. For $a<b<c \in \mathbb{R}_{+}$

$$
F_{a}^{c}(b) \cdot J_{a}^{c}=\left(J_{a}^{b} \otimes J_{b}^{c}\right) \Delta .
$$

Proof. Expressing the simplex

$$
\Delta_{a}^{c}(n)=\left\{\left(s_{1}, s_{2}, \ldots, s_{n}\right) \in \mathbb{R}_{+}^{n}: a<s_{1}<s_{2}<\cdots<s_{n}<c\right\}
$$

as the disjoint union of Cartesian products

$$
\Delta_{a}^{c}(n)=\bigcup_{j=0}^{n}\left(\Delta_{a}^{b}(j) \times \Delta_{b}^{c}(n-j)\right),
$$

where, by definition, the Cartesian product of a set with $\Delta_{a}^{b}(0)$ or with $\Delta_{b}^{c}(0)$ is itself, we have for arbitrary $n \in \mathbb{N}$ and $\alpha_{1}, \alpha_{2}, \ldots, \alpha_{n}, \beta_{1}, \beta_{2}, \ldots, \beta_{n} \in\{0,1,2, \ldots, N\}$

$$
\begin{aligned}
& F_{a}^{c}(b) J_{a}^{c}\left(d \Lambda_{\alpha_{1}}^{\beta_{1}} \otimes d \Lambda_{\alpha_{2}}^{\beta_{2}} \otimes \ldots \otimes d \Lambda_{\alpha_{n}}^{\beta_{n}}\right) \\
= & F_{a}^{c}(b) \int_{a<s_{1}<s_{2}<\cdots<s_{n}<c} d \Lambda_{\alpha_{1}}^{\beta_{1}}\left(s_{1}\right) d \Lambda_{\alpha_{2}}^{\beta_{2}}\left(s_{2}\right) \ldots d \Lambda_{\alpha_{n}}^{\beta_{n}}\left(s_{n}\right) \\
= & F_{a}^{c}(b) \int_{\left(s_{1}, s_{2}, \ldots, s_{n}\right) \in \Delta_{a}^{c}(n)} d \Lambda_{\alpha_{1}}^{\beta_{1}}\left(s_{1}\right) d \Lambda_{\alpha_{2}}^{\beta_{2}}\left(s_{2}\right) \ldots d \Lambda_{\alpha_{n}}^{\beta_{n}}\left(s_{n}\right) \\
= & F_{a}^{c}(b) \int_{\left(s_{1}, s_{2}, \ldots, s_{n}\right) \in \cup_{j=0}^{n}\left(\Delta_{a}^{b}(j) \times \Delta_{b}^{c}(n-j)\right)} d \Lambda_{\alpha_{1}}^{\beta_{1}}\left(s_{1}\right) d \Lambda_{\alpha_{2}}^{\beta_{2}}\left(s_{2}\right) \ldots d \Lambda_{\alpha_{n}}^{\beta_{n}}\left(s_{n}\right) \\
= & \sum_{j=0}^{n} \int_{\left(s_{1}, s_{2}, \ldots, s_{j}\right) \in \Delta_{a}^{b}(j)} d \Lambda_{\alpha_{1}}^{\beta_{1}}\left(s_{1}\right) d \Lambda_{\alpha_{2}}^{\beta_{2}}\left(s_{2}\right) \ldots d \Lambda_{\alpha_{j}}^{\beta_{j}}\left(s_{j}\right) \\
= & \sum_{\left(s_{j+1}, s_{j+2}, \ldots, s_{n}\right) \in \Delta_{b}^{c}(n-j)}^{n} d \Lambda_{\alpha_{1+1}}^{\beta_{j+1}}\left(s_{j+1}\right) d \Lambda_{\alpha_{j+2}}^{\beta_{j+2}}\left(s_{j+2}\right) \ldots d \Lambda_{\alpha n}^{\beta n}\left(s_{n}\right) \\
& d \Lambda_{\alpha_{1}}^{\beta_{1}}\left(s_{1}\right) d \Lambda_{\alpha_{2}}^{\beta_{2}}\left(s_{2}\right) \ldots d \Lambda_{\alpha_{j}}^{\beta j}\left(s_{j}\right) \\
& \int_{a<s_{1}<s_{2}<\cdots<s_{j}<b}
\end{aligned}
$$


From ALGEBraic to ANALYTIC DOUble PRODUCT INTEGRALS.

$$
\begin{aligned}
& \otimes \int_{b<s_{j+1}<s_{j+2}<\cdots<s_{n}<c} d \Lambda_{\alpha_{1+1}}^{\beta_{j+1}}\left(s_{j+1}\right) d \Lambda_{\alpha_{j+2}}^{\beta_{j+2}}\left(s_{j+2}\right) \ldots d \Lambda_{\alpha n}^{\beta n}\left(s_{n}\right) \\
= & \sum_{j=0}^{n} J_{a}^{b}\left(d \Lambda_{\alpha_{1}}^{\beta_{1}} \otimes d \Lambda_{\alpha_{2}}^{\beta_{2}} \otimes \ldots \otimes d \Lambda_{\alpha_{j}}^{\beta_{j}}\right) \otimes \ldots \ldots J_{b}^{c}\left(d \Lambda_{\alpha_{j+1}}^{\beta_{j+1}} \otimes d \Lambda_{\alpha_{j+2}}^{\beta_{j+2}} \otimes \ldots \otimes d \Lambda_{\alpha_{n}}^{\beta_{n}}\right) \\
= & \left(J_{a}^{b} \otimes J_{b}^{c}\right) \Delta\left(d \Lambda_{\alpha_{1}}^{\beta_{1}} \otimes d \Lambda_{\alpha_{2}}^{\beta_{2}} \otimes \ldots \otimes d \Lambda_{\alpha_{n}}^{\beta_{n}}\right) .
\end{aligned}
$$

The result now follows by linearity of both sides of (6)

\section{Double Product integrals in Fock SPACE.}

Given intervals $a<b$ and $c<d$ in $\mathbb{R}_{+}$and a double product such as $R[h]=\vec{\prod}$ $(1+d r[h])$ where the coefficients of $d r[h]$ belong to the algebra $\mathcal{L}$ of Itô differentials we may try to construct a corresponding operator on the exponential domain in the double Fock space

$$
\mathcal{F}\left(L^{2}\left(\mathbb{R}_{+} ; \mathbb{C}^{N}\right)\right) \otimes \mathcal{F}\left(L^{2}\left(\mathbb{R}_{+} ; \mathbb{C}^{N}\right)\right)=\mathcal{F}\left(L^{2}\left(\mathbb{R}_{+} ; \mathbb{C}^{N}\right) \oplus L^{2}\left(\mathbb{R}_{+} ; \mathbb{C}^{N}\right)\right)
$$

by applying the homomorphism $J_{a}^{b} \otimes J_{c}^{d}$ to the coefficients of the formal power series $R[h]$ and replacing $h$ by a complex variable $z$. Assuming convergence we may thus define a family of operators on the exponential domain

$$
R_{a, c}^{b, d}(z)=\prod_{(s, t) \in] a, b] \times] c, d]}(1+r(z ; d s, d t))=\left.\left(J_{a}^{b} \otimes J_{c}^{d}\right) \vec{\prod}(1+d r[h])\right|_{h=z}
$$

In view of Theorem 3 and Theorem 6 we should expect these to satisfy

$$
R_{a, c}^{b, d}(z)=R_{a, c}^{x, d}(z) R_{x, c}^{b, d}(z)=R_{a, y}^{b, d}(z) R_{a, c}^{b, y}(z), a<x<b, c<y<d,
$$

at least in the weak sense where the operator products are circumvented by moving the first operator to its adjoint in exponential matrix elements, that is, for arbitrary exponential vectors $e(f), e(g), e(p), e(q)$

$$
\begin{aligned}
\left\langle e(f) \otimes e(g), R_{a, c}^{b, d}(z) e(p) \otimes e(q)\right\rangle & =\left\langle\left(R_{a, c}^{x, d}(z)\right)^{\dagger} e(f) \otimes e(g), R_{x, c}^{b, d}(z) e(p) \otimes e(q)\right\rangle \\
& =\left\langle\left(R_{a, y}^{b, d}(z)\right)^{\dagger} e(f) \otimes e(g), R_{a, c}^{b, y}(z) e(p) \otimes e(q)\right\rangle,
\end{aligned}
$$

as well as

$$
\left(\prod_{(s, t) \in] a, b] \times] c, d]}^{\rightarrow \leftarrow}(1+r(z ; d s, d t))\right)^{\dagger}=\varlimsup_{(s, t) \in] a, b] \times] c, d]}^{\leftarrow \rightarrow}\left(1+r^{\dagger}(\bar{z} ; d s, d t)\right)
$$




$$
\left(\prod_{(s, t) \in] a, b] \times] c, d]}^{\rightarrow \leftarrow}(1+r(z ; d s, d t))\right)^{-1}=\prod_{(s, t) \in] a, b] \times] c, d]}^{\leftarrow}\left(1+r^{\prime}(z ; d s, d t)\right)
$$

where $r^{\dagger}[h]$ is formed by appling the tensor product involution to the coefficients of $r[h]$ and $r^{\prime}[h]$ is the quasiinverse of $r[h]$, the latter again at least in the weak sense that

$$
\begin{aligned}
& \langle e(f) \otimes e(g), e(p) \otimes e(q)\rangle \\
= & \left\langle\prod_{(s, t) \in] a, b] \times] c, d]}^{\leftarrow \rightarrow}\left(1+r^{\dagger}(\bar{z} ; d s, d t)\right) e(f) \otimes e(g), \prod_{(s, t) \in] a, b] \times] c, d]}^{\leftarrow \rightarrow}\left(1+r^{\prime}(z ; d s, d t)\right) e(p) \otimes e(q)\right\rangle \\
= & \left\langle\prod_{(s, t) \in] a, b] \times] c, d]}^{\rightarrow \leftarrow}\left(1+r^{\prime \dagger}(z ; d s, d t)\right) e(f) \otimes e(g), \prod_{(s, t) \in] a, b] \times] c, d]}^{\leftarrow}(1+r(z ; d s, d t)) e(p) \otimes e(q)\right\rangle .
\end{aligned}
$$

However an analytic theory of double product integrals in Fock space of this kind is obstructed by divergence problems. It is evident that if the formal power series $r[h]$ has many nonzero coefficients these may be of a novel and possibly intractable kind. But even if we make the simpifying assumption that only the first order coefficient $r_{1}$ is nonzero (which we do henceforth) divergence problems remain. For example the (commutative) double product $\prod(1+z d \Lambda \otimes d \Lambda)$, where $\Lambda$ is the conservation process of one-dimensional quantum stochastic calculus is divergent for all nonzero values of $z$ in the sense that for arbitrary $f$ and $g$ non-zero on $] a, b]$ and $f^{\prime}, g^{\prime}$ non zero on $\left.] c, d\right]$, the exponential matrix element

$$
\left\langle e(f) \otimes e\left(f^{\prime}\right), \prod_{(s, t) \in] a, b] \times] c, d]}(1+z d \Lambda(s) \otimes d \Lambda(t)) e(g) \otimes e\left(g^{\prime}\right)\right\rangle
$$

has zero radius of convergence in $z$. On the other hand it is clear (since $(d A)^{2}=0$ ) that $\prod_{(s, t) \in] a, b] \times] c, d]}(1+z d A(s) \otimes d A(t))$ exists in this sense and is given by $\exp \left(a\left(\chi_{a}^{b}\right) \otimes a\left(\chi_{c}^{d}\right)\right)$ where $a\left(\chi_{a}^{b}\right)$ is the annihilation operator corresponding to the indicator function of the interval $] a, b]$. More interestingly the double product $\prod_{(s, t) \in] a, b] \times] c, d]}\left(1+z\left(d A^{\dagger}(s) \otimes\right.\right.$ $d A(t)-d A(s) \otimes d A^{\dagger}(t)$ ) exists (for all real $z$ ) as a unitary operator satisfying $(7)$ [7],[8]. It is conjectured that $\prod_{(s, t) \in] a, b] \times] c, d]}\left(1+z\left(d \Lambda(s) \otimes d A(t)-d \Lambda(s) \otimes d A^{\dagger}(t)\right)\right.$ exists and continues to satisfy the quantum Yang-Baxter equation in $\mathcal{F}\left(L^{2}\left(\mathbb{R}_{+} ; \mathbb{C}^{N}\right)\right) \otimes$ $\mathcal{F}\left(L^{2}\left(\mathbb{R}_{+} ; \mathbb{C}^{N}\right)\right) \otimes \mathcal{F}\left(L^{2}\left(\mathbb{R}_{+} ; \mathbb{C}^{N}\right)\right)$.

\section{REFERENCES}

[1] N Bourbaki, Algebra I, Addison-Wesley (1974), Chapter III Section 11.5, Lemma 1. 
[2] B Enriquez, Quantization of Lie bialgebras and shuffle algebras of Lie algebras, Selecta Mathematica (new series) 7 321-407 (2001).

[3] P Etingof and O Schiffman Lectures on quantum groups, International Press (1998).

[4] T M W Eyre, Quantum stochastic calculus and representations of Lie bialgebras, Springer lecture Notes in Mathematics 1692 (1998).

[5] R L Hudson, Itô calculus and quantisation of Lie bialgebras, Annales de l'Institue H Poicaré-PR 41 (P-A Meyer memorial volume), 375-390 (2005).

[6] R L Hudson, The method of double product integrals in quantisation of Lie bialgebras, pp303-319, in Quantum Probability and Infinite Dimensional Analysis from Foundations to Applications, Proceedings, Greifswald 2003, eds M Schürmann et al, World Scientific (2005).

[7] R L Hudson, An analytic double product integral, to appear in Proceedings of 2005 QP workshop.

[8] R L Hudson, A double dilation constructed from a double product of rotations, to appear in Markov Processes and Applications ( $\mathrm{J}$ T Lewis mmemorial volume, 2007).

[9] R L Hudson and S Pulmannová, Double product integrals and Enriquez quantisation of Lie bialgebras I: The quasitriangularity relations, Journal of Mathematical Physics 45, 2090-2105 (2004).

[10] R L Hudson and S Pulmannová, Double product integrals and Enriquez quantisation of Lie bialgebras II: The quantum Yang-Baxter equation, Letters in Mathematical Physics 72, 211-224 (2005).

[11] R L Hudson and S Pulmannová, Chaotic expension of elements of the universal enveloping algebra of a Lie algebra associated with a quantum stochastic calculus, Proceedings of the London Mathematical Society (3rd Series) 77, 462-480 (1998).

[12] S Majid, Foundations of quantum group theory, Cambridge University press (1995).

[13] P-A Meyer, Quantum probability for probabilists, Springer lecture Notes in Mathematics 1538 (1993)

[14] K R Parthasarathy, An introduction to quantum stochastic calculus, Birkhäuser (1992) 